\title{
SRPK1/2 and PP1a exert opposite functions by modulating SRSF1-guided MKNK2 alternative splicing in colon adenocarcinoma
}

Hongda Liu', Zheng Gong ${ }^{2}$, Kangshuai Li $i^{3}$, Qun Zhang ${ }^{4}$, Zekuan $X u^{1 *}$ and Yunfei $\mathrm{Xu}^{3^{*}}$

\begin{abstract}
Background: The Mnk2 kinase, encoded by MKNK2 gene, plays critical roles in MAPK signaling and was involved in oncogenesis. Human MKNK2 pre-mRNA can be alternatively spliced into two splicing isoforms, the MKNK2a and MKNK2b, thus yielding Mnk2a and Mnk2b proteins with different domains. The involvement of Mnk2 alternative splicing in colon cancer has been implicated based on RNA-sequencing data from TCGA database. This study aimed at investigating the upstream modulators and clinical relevance of Mnk2 alternative splicing in colon adenocarcinoma (CAC).

Methods: PCR, western blotting and immunohistochemistry $(\mathrm{IHC})$ were performed to assess the expression of Mnk2 and upstream proteins in CAC. The function of Mnk2 and its regulators were demonstrated in different CAC cell lines as well as in xenograft models. Two independent cohorts of CAC patients were used to reveal the clinical significance of MKNK2 alternative splicing.

Results: Comparing with adjacent nontumorous tissue, CAC specimen showed a decreased MKNK2a level and an increased MKNK2b level, which were correlated with KRAS mutation and tumor size. The SRSF1 (serine/arginine-rich splicing factor 1) was further confirmed to be the major splicing factor targeting MKNK2 in CAC cells. Higher expression of SRPK1/2 or decreased activity of PP1a were responsible for enhancing SRSF1 phosphorylation and nucleus translocation, subsequently resulted in a switch of MKNK2 alternative splicing.

Conclusions: Our data showed that phosphorylation and subcellular localization of SRSF1 were balanced by SRPK1/ 2 and PP1a in CAC cells. High nucleus SRSF1 promoted MKNK2 splicing into MKNK2b instead of MNK2a, consequently enhanced tumor proliferation.
\end{abstract}

Keywords: Alternative splicing, Colon adenocarcinoma, MKNK2, SRSF1, SRPK1/2

\footnotetext{
*Correspondence: xuzekuan@njmu.edu.cn; xuyunfei1988@126.com

'Department of General Surgery, The First Affiliated Hospital of Nanjing Medical University, 300 Guangzhou Road, Nanjing 210029, China

${ }^{3}$ Department of General Surgery, Qilu Hospital of Shandong University, Jinan 250012, China

Full list of author information is available at the end of the article
}

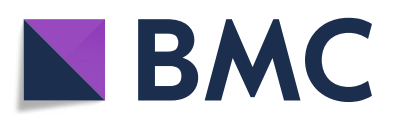

(- The Author(s). 2021 Open Access This article is licensed under a Creative Commons Attribution 4.0 International License, which permits use, sharing, adaptation, distribution and reproduction in any medium or format, as long as you give appropriate credit to the original author(s) and the source, provide a link to the Creative Commons licence, and indicate if changes were made. The images or other third party material in this article are included in the article's Creative Commons licence, unless indicated otherwise in a credit line to the material. If material is not included in the article's Creative Commons licence and your intended use is not permitted by statutory regulation or exceeds the permitted use, you will need to obtain permission directly from the copyright holder. To view a copy of this licence, visit http://creativecommons.org/licenses/by/4.0/. The Creative Commons Public Domain Dedication waiver (http://creativecommons.org/publicdomain/zero/1.0/) applies to the data made available in this article, unless otherwise stated in a credit line to the data. 


\section{Background}

Colorectal cancer ranks the third on both malignant morbidity and cancer-related deaths worldwide [1]. Approximately $20 \%$ of colorectal cancer patients were diagnosed at an extensive-stage with distant distribution, and the 5-year survival rate is less than 15\% [2]. As the most common histological subtype, colon adenocarcinoma (CAC) can be treated with surgical resection, adjuvant chemotherapy, and radiation treatment. Unfortunately, molecular understanding of CAC progression remains limited although its incidence and mortality rates have been declined during the past decades [3].

Numerous well-known kinase networks are considered to play roles in CAC pathogenesis, such as EGFR, MAPKs, c-Src, et al. The MAP kinase-interacting serine/ threonine-protein kinases (Mnks), downstream of MAPKs, are protein kinases that can phosphorylate eIF4E and enhance oncogenic mRNA translation [4]. There are two members of Mnk family in human cells: Mnk1 and Mnk2. We have previously reported the clinical significance of Mnk1 in epithelial ovarian cancer [5]. Mnk2 has also been reported to be involved in malignancies such as prostate cancer and lung cancer [6, 7]. Mnk2a and Mnk2b are two protein isoforms that derived from the $M K N K 2$ pre-mRNA by alternative splicing, namely $M K N K 2 a$ and $M K N K 2 b$, respectively [8]. Mnk2a contains a MAPK-binding site in its C-terminus while Mnk2b lacks it [9]. The specific role of Mnk2a seems ambiguous in tumorigenesis. On one hand, hyperactivation of Mnk2a can phosphorylate eIF4E thus exerts oncogenic effects [9]. One the other hand, Mnk2a directly interacts and phosphorylates p38 $\alpha$-MAPK, subsequently inducing cell death and suppressing Ras-induced transformation [10]. In contrast, Mnk2b is generally recognized as a pro-oncogenic kinase due to its deficiency in binding p38 $\alpha$-MAPK but reserves capacity of phosphorylating eIF4E [10].

According to TCGA database, the MKNK2a/MKNK2b ratio was reported to be downregulated in CAC tissues compared with nontumorous colon tissues [10], however its clinical significance and upstream regulators remain unknown. Here we discovered that SRSF1 (Serine and Arginine Rich Splicing Factor 1), an essential sequence specific splicing factor involved in pre-mRNA splicing, is the predominant splicing factor that promotes synthesis of $M K N K 2 b$ instead of MKNK2a. Interestingly, SRSF1guided MKNK2a-MKNK2b switch is dependent on its phosphorylation and subsequent nucleus transportation. Our data further revealed the antergic effects of SRPK1/ 2 (SR-specific protein kinase) and protein phosphatase $1 \alpha(\mathrm{PP} 1 \alpha)$ on directly modulating SRSF1 phosphorylation. Besides, SRPK1/2 and nucleus SRSF1 were verified to be independent prognostic factors for the clinical outcome of CAC patients from two retrospective cohorts.
Finally, the tumor-related role of SRPK1/2, SRSF1, Mnk2b, and PP1 $\alpha$ were validated in CAC cell lines as well as in xenograft models.

\section{Methods \\ Cells and reagents}

Human CAC cell lines including Caco-2, HT-29, HCT116, SW480 and SW620 were purchased from the American Type Culture Collection (ATCC, Manassas, VA, USA). Nontumorous NCM460 colon epithelial cells were obtained from Jennio Biotechnology (Guangzhou, China). Cells were cultured in DMEM supplemented with $10 \%$ fetal bovine serum (FBS) and 1\% penicillin/ streptomycin under $5 \% \mathrm{CO} 2$ at $37{ }^{\circ} \mathrm{C}$ atmosphere. The chemicals used in our study were listed in Supplementary Table S6.

\section{DNA constructs, siRNAs, shRNAs}

pCDNA3.1-vector was obtained from Invitrogen. Fulllength of KRAS-G12V cDNA was cut from pBabe K-Ras $12 \mathrm{~V}$ vector (Cat. \#12544, Addgene) and cloned into pcDNA3.1-vector (Invitrogen) via BamH1 and Xba1 restriction sites, thus generating the pCDNA3.1-RASG12V construct. Other DNA constructs including pCDNA3.1-Mnk2a, pCDNA3.1-Mnk2b, pCDNA3.1SRPK1-WT, pCDNA3.1-SRPK1-K109A, pCDNA3.1SRPK2-WT, pCDNA3.1-SRPK2-K110A, pCDNA3.1PP1 $\alpha-W T$, pCDNA3.1-PP1 $\alpha-T 320 A$, and pCDNA3.1PP1 $\alpha-\mathrm{H} 125 \mathrm{~A}$ were generated by GenePharma (Shanghai, China). The siRNAs and shRNAs were listed in Supplementary Table S6.

Transient transfections were conducted by using Lipofectamine3000 Transfection Reagent (Cat. \# L3000015, Thermo Fisher Scientific, Pittsburgh, PA, USA) according to the manufacturer's instructions. Puromycin was used to select and maintain stable overexpression cells. Lentivirus infection of SRPK1 and SPRK2 shRNAs were conducted as previously described [11].

\section{Patients and tissue samples}

Two independent cohorts of patients were retrospectively enrolled in this study. The primary cohort enrolled 32 CAC patients who underwent surgical treatment in Qilu Hospital of Shandong University (Jinan, China) from 2016 to 2017. The fresh-resected carcinoma tissues and paired adjacent nontumorous colon tissues were all frozen in liquid-nitrogen until experimental test. The validation cohort comprised of $100 \mathrm{CAC}$ cases that underwent tumor resection in 2007, and the tissue specimens were formalin-fixed paraffin-embedded (\#HColA180Su17, Shanghai Outdo Biotech, Shanghai, China). The patients in validation cohort were followed up until July 2015 (ranging 2-97 months). All the patients in primary cohort and validation cohort underwent R0 
resection and classified with TNM stage I-IIIC at the time of surgical intervention according to the 7th AJCC/ UICC TNM classification system. None of the patients in the two cohorts received any adjunctive therapy before tissue sample collection. The clinicopathological characteristics of the patients in two cohorts were summarized in Supplemental Table S1 and Supplemental Table S3, respectively. Informed consents were obtained from all patients or immediate relatives. All experiments were approved and supervised by the Ethics Committee of Qilu Hospital of Shandong University.

\section{Real-time PCR (RT-PCR) and real-time quantitative PCR (RT-qPCR)}

Paired tumor and nontumorous specimens from primary cohort were subjected for RNA isolation and RT-qPCR using GAPDH as internal control. Briefly, total RNA was extracted from tissues and cells using TRIzol reagent. RNA was reversely transcribed into cDNA by PrimeScript RT Master Mix (TaKaRa, Kyoto, Japan). Quantitative polymerase chain reaction (qPCR) was conducted with SYBR Premix Ex Taq II kits (TaKaRa, Kyoto, Japan) as described before [12]. RT-PCR was performed using the one step RT-PCR kit (QIAGEN) following the manufacturer's protocol. The primers used for RT-PCR and RT-qPCR were listed in Supplemental Table S6.

\section{Cell proliferation and colony formation}

The proliferation assay colony formation assay were conducted as previously described [13, 14]. Proliferation capacity was assessed by 3-(4,5-dimethylthiazol-2-yl)-2, 5-diphenyl-2H-tetrazolium bromide (MTT) assays at designated time points $(6 \mathrm{~h}, 24 \mathrm{~h}, 48 \mathrm{~h}, 72 \mathrm{~h}, 96 \mathrm{~h})$. Colonies were tested after incubation for 10 days in DMEM.

\section{RNA-binding protein immunoprecipitation (RIP)}

The RIP assay was conducted by using an RNA-Binding Protein Immunoprecipitation Kit (Millipore) according to the manufacture's procedure. The binding between the SRSF1 protein and $M K N K 2 a / 2 b$-mRNA was determined by RT-PCR and Western blotting of immunoprecipitated mixture.

\section{Sample lysis and immunoblotting}

Tissue samples or cultured cells were lysed using RIPA lysis buffer (Cat. \#P0013, Beyotime, Beijing, China) containing protease and phosphatase inhibitors for total protein test. For nucleus isolation, cells were processed using the Nucleus and Cytosol Protein Extraction Kit (Cat. \#P0027, Beyotime) according to the manufacture's instruction. The immunoblotting was conducted as described previously [14]. The antibodies used in our study were listed in Supplementary Table S6 at 1:1000 dilution.

\section{Immunohistochemical (IHC) staining}

The IHC staining was performed as described before [14] using anti-SRSF1, anti-SRPK1, anti-SRPK2, antiPP1 $\alpha$, anti-PP1 $\alpha-$ - $-T h r 320$ antibodies (Table S6) at 1 : 300 dilution. The staining results were semi-quantified by multiplying the positive percentage score ( 0 , no positive stained cells; $1,0-25 \%$ positive; $2,26-50 \%$ positive; $3,51-75 \%$ positive; $4,76-100 \%$ positive) and staining intensity score ( 0 , negative staining; 1 , slightly yellow; 2 , dark yellow; 3, yellow brown), ranging $0-12$. As for the quantification of SRSF1 staining, the nucleus staining and cytosol staining were scored separately. Either the nucleus staining or the cytosol staining were scored as $0-12$ as described above. The cut-off of IHC scores were obtained by using receiver operating characteristic (ROC) curves to distinguish high- or low-expression groups.

\section{Immunoprecipitation}

Transfected cells were harvested and lysed with RIPA buffer. After protein quantification using the BCA kit (Thermo Fisher Scientific), equal amount of lysates were incubated with HA agarose beads overnight an $4{ }^{\circ} \mathrm{C}$. The immunoprecipitated mixture were subjected to Western blotting after washing out the nonspecific bindings with RIPA buffer.

\section{GST-pull down}

The purified GST protein was obtained from Prof. Jinpeng Sun in Shandong University (Jinan, China). The purified GST-tagged SRPK1 and GST-tagged SRPK2 proteins were purchased from Novus Biologicals (CO, USA). The His-SRSF1 protein was purchased from Creative Biomart (NY, USA). The GST-pull down assay was conducted as described before using GST agarose beads [15].

\section{In vitro phosphorylation test}

Purified SRPK1 or SRPK2 proteins were incubated with SRSF1 proteins in kinase reaction buffer $(25 \mathrm{mM}$ Tris, $\mathrm{pH} 7.5$, with $10 \mathrm{mM} \mathrm{MgCl} 2,2 \mathrm{mM}$ DTT, $5 \mathrm{mM} \beta$ Glycerolphosphate, $0.1 \mathrm{mM} \mathrm{Na} 3 \mathrm{VO} 4$, and $2 \mathrm{mM}$ EGTA) containing $20 \mu \mathrm{M}$ ATP for $30 \mathrm{~min}$ at $37^{\circ} \mathrm{C}$ water bath. Reactions were then quenched by adding SDS loading buffer and subjected to western blot assay.

\section{Mice model and xenograft}

Transfected SW480 cells $\left(4 \times 10^{6}\right)$ were subcutaneously injected into six-week male Balb/c nude mice. Each group contained 5 mice. Tumor size was measured every 5 days using the following formula: $\mathrm{V}=\left(\right.$ length $\times$ width $\left.^{2}\right) /$ 2. The mice were sacrificed on the 21st day after injection, and the xenograft tumors were removed and weighed as described before [13]. The Animal Welfare 
Committee of Shandong University approved all procedures involving animals.

\section{Statistics}

Data are presented as the mean \pm standard deviation (SD). Statistical analyses were conducted using SPSS 20.0 Software. Correlations between mRNAs were analyzed using Spearman correlation test. Correlations between proteins and patients' characteristics were analyzed using Chi-square test. Overall survival (OS) was assessed using the Kaplan-Meier method and significance was analyzed using the log-rank test. Multivariate cox regression analyses were carried out to identify independent prognostic factors. For the cellular and xenograft data, the difference between two groups was tested using Student's t-test, while One-way ANOVA analysis was used to compare data among more than two groups. A value of $P<0.05$ indicated a statistically significant result.

\section{Results \\ MKNK2 alternative splicing is altered in CAC tissues and affected by KRAS mutation}

$M K N K 2$ can be alternatively spliced to $M K N K 2 a$ and $M K N K 2 b$ mRNAs and consequently translated into two protein isoforms: Mnk2a and Mnk2b (Fig. 1a). By analyzing the mRNAs level of $M K N K 2$ isoforms in 32 paired tissues (Table S1), we found that $M K N K 2 a$ was downregulated in CAC tumor tissues while $M K N K 2 b$ was upregulated (Fig. 1b). Mutations of KRAS and TP53 are the most well-characterized onco-driver of $\mathrm{CAC}$, and we next investigated whether they have effects on MKNK2 alternative splicing (Fig. 2c). Although TP53 seemed to have no impact on $M K N K 2$ mRNA levels, MKNK2a splicing was inhibited while $M K N K 2 b$ was remarkably upregulated in KRAS-mutated CAC samples. Therefore, we enrolled both KRAS-WT cells (Caco-2 and HT-29) and KRAS-mutated cells (HCT-116 and SW480) to conduct cellular validation. Comparing with Caco-2 and HT-29 cells, HCT-116 and SW480 cells showed lower $M K N K 2 a$ but higher MKNK2b mRNA levels (Fig. 1d). Furthermore, transfecting Caco-2/HT-29 cells with $K R A S-\mathrm{G} 12 \mathrm{~V}$ plasmids significantly altered the predominant MKNK2 isoform from $M K N K 2 a$ to $M K N K 2 b$ (Fig. $1 \mathrm{e})$, indicating that $K R A S$ can affect $M K N K 2$ alternative splicing. We next aimed to seek whether the KRAS$\mathrm{G} 12 \mathrm{~V}$-induced $M K N K 2 b$ was resulted from increased RNA synthesis or decreased RNA degradation using NCM460 cells. Actinomycin D is a potent inhibitor that can inhibit poly(A)RNA synthesis in a dose-dependent manner [16]. According to our data, actinomycin D treatment significantly attenuated the KRAS-G12V-induced $M K N K 2 b$ expression at $20 \mathrm{nM}$ or higher concentrations (Fig. 1f), implying this $M K N K 2 b$ alteration was correlated with RNA synthesis instead of RNA degradation. Interestingly, $M K N K 2 a$ and $M K N K 2 b$ showed opposite effects on KRAS-G12V-induced colony formation of NCM460 cells (Fig. 1g), indicating their distinct roles in modulating tumor development.

\section{MKNK2 alternative splicing is correlated with tumor growth instead of LN metastasis}

Additionally, $M K N K 2 a$ was relatively lower in tumors with larger size while $M K N K 2 b$ was higher in larger CAC tissues (Fig. 1h). Spearman correlation test showed that tumor diameter was negatively correlated to $M K N K 2 a$ level and positively correlated to $M K N K 2 b$ level (Fig. S1A, S1B). Apparently, the MKNK2al $M K N K 2 b$ ratio was decreased in tumor specimens with larger diameter (Fig. S1C). In contrast, we didn't identify any significant correlation between $M K N K 2$ mRNA levels with lymph node (LN) metastasis (Figure S1D), indicating MKNK2 may have little effect on modulating tumor invasion. Since we showed that $M K N K 2$ alternative splicing is closely correlated with KRAS mutation, we chose HCT-116 and SW480 cell for following cellular tests in this study. Although transfection of Mnk2a had no obvious effect on CAC cell proliferation (Fig. 1i) nor colony formation (Fig. 1j), overexpressing Mnk2b significantly enhanced cell proliferation capacity. Both our clinical and cellular findings suggested a novel tumor-promoting role of Mnk2b on CAC progression.

\section{Nucleus SRSF1 is closely correlated with MKNK2 alternative splicing in tumor tissues}

Since KRAS is not a splicing factor and is less possible for directly regulating $M K N K 2$ alternative splicing, we next aimed to figure out the upstream splicing factors targeting $M K N K 2$. Serine/arginine-rich splicing factors (SRSFs) are the major splicing factors that regulate alternative splicing process in mammalian cells [17]. By testing the mRNA levels of $S R S F$ family members, we found that SRSF2 showed a positive relationship with $M K N K 2 b$ level. In contrast, SRSF3, SRSF4 and SRSF9 showed negative correlations with $M K N K 2 a$ level (Figure S2, Table S2). However, only SRSF1 was negatively correlated with $M K N K 2 a$ and positively correlated with $M K N K 2 b$ simultaneously, indicating its predominant role on modulating MKNK2a-MKNK2b switch in CAC tissues (Fig. 2a, b). Moreover, RNA-immunoprecipitation results confirmed the interaction between SRSF1 protein and $M K N K 2 a / 2 b$-mRNA in SW480 cells (Fig. 2c).

Therefore, we further investigated whether SRSF1 participate in CAC progression via MKNK2a-MKNK2b switch. RT-qPCR and immunoblotting data showed that SRSF1 was upregulated in tumor tissues compared with adjacent nontumorous tissues on both RNA and protein levels (Fig. 2d, e). Interestingly, IHC data identified a 


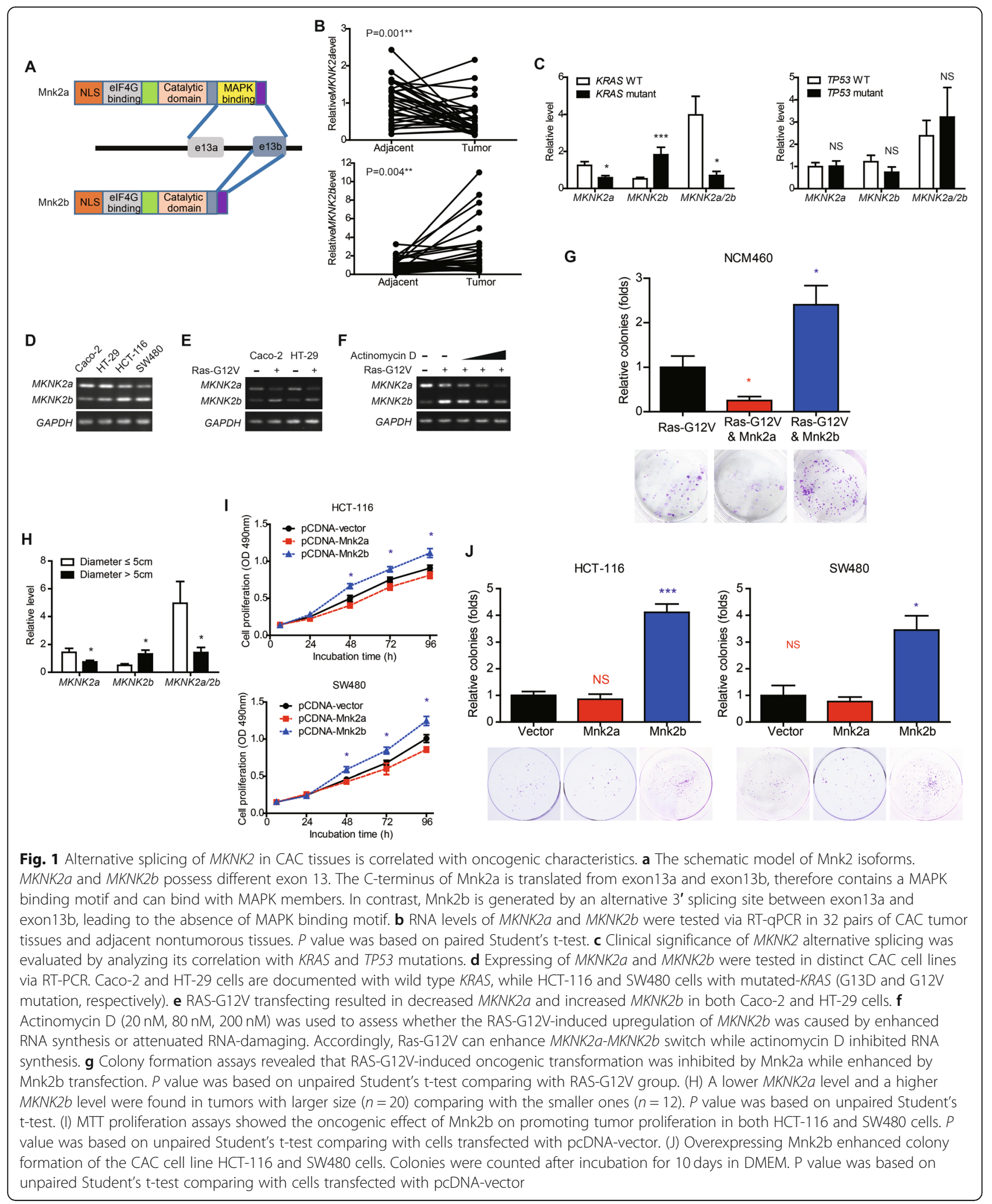

diffused staining of SRSF1 protein in both nucleus and cytoplasm (Fig. 2f). To better evaluate the alteration of SRSF1 in CAC tissues, we separately assessed its expression in nucleus or cytoplasm. No statistical difference was observed between tumor tissues and adjacent tissues regarding to cytoplasm-SRSF1. Nevertheless, 


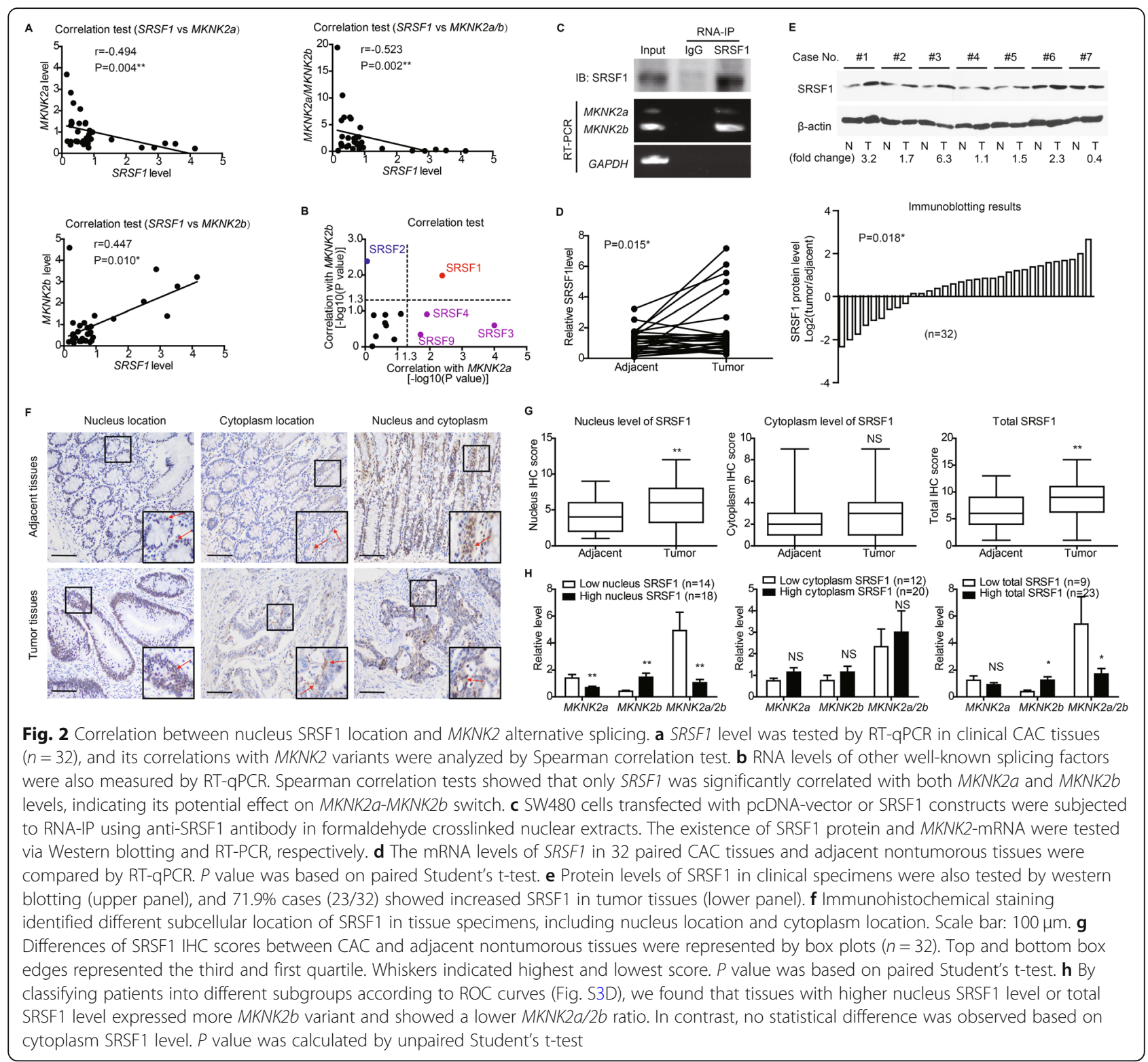

CAC samples showed higher total SRSF1 and nucleusSRSF1 than nontumorous tissues (Fig. 2g). In addition, both total SRSF1 and nucleus-SRSF1 were positively correlated to $M K N K 2 b$-mRNA level, while negatively correlated to $M K N K 2 a$ and $M K N K 2 a / M K N K 2 b$ (Figure S3A, S3B). In contrast, cytoplasm-SRSF1 showed no significant correlation with MKNK2 alternative splicing (Figure S3C). Using ROC method (Figure S3D), we next divided the primary cohort into subgroups based on SRSF1 levels in different subcellular locations. Consistent with Spearman correlation test, Chi-square results also revealed a significant difference of $M K N K 2$ alternative splicing between low nucleus-SRSF1 and high nucleus-SRSF1 groups (Fig. 2h). Similar results were obtained based on total SRSF1 expression but not cytoplasm-SRSF1 (Fig. 2h).
TNPO3-dependent nucleus transportation of SRSF1 determines MKNK2 alternative splicing and cell proliferation

We set out to explore the possible effects of SRSF1 on $M K N K 2$ alternative splicing in CAC cells. Knockdown of SRSF1 can significantly inhibit $M K N K 2 a-M K N K 2 b$ switch according to RT-PCR (Fig. 3a) and RT-qPCR data (Fig. 3b), respectively. Of note, cell proliferation and colony formation capacities were also attenuated by silencing SRSF1 (Fig. 3c, d). On the other hand, overexpressing SRSF1 resulted in MKNK2a-MKNK2b switch and enhanced cell proliferation (Fig. 3e-h). Taking into consideration that only nucleus-SRSF1, instead of cytoplasm-SRSF1, showed a significant correlation with $M K N K 2$ alternative splicing in CAC samples, we 


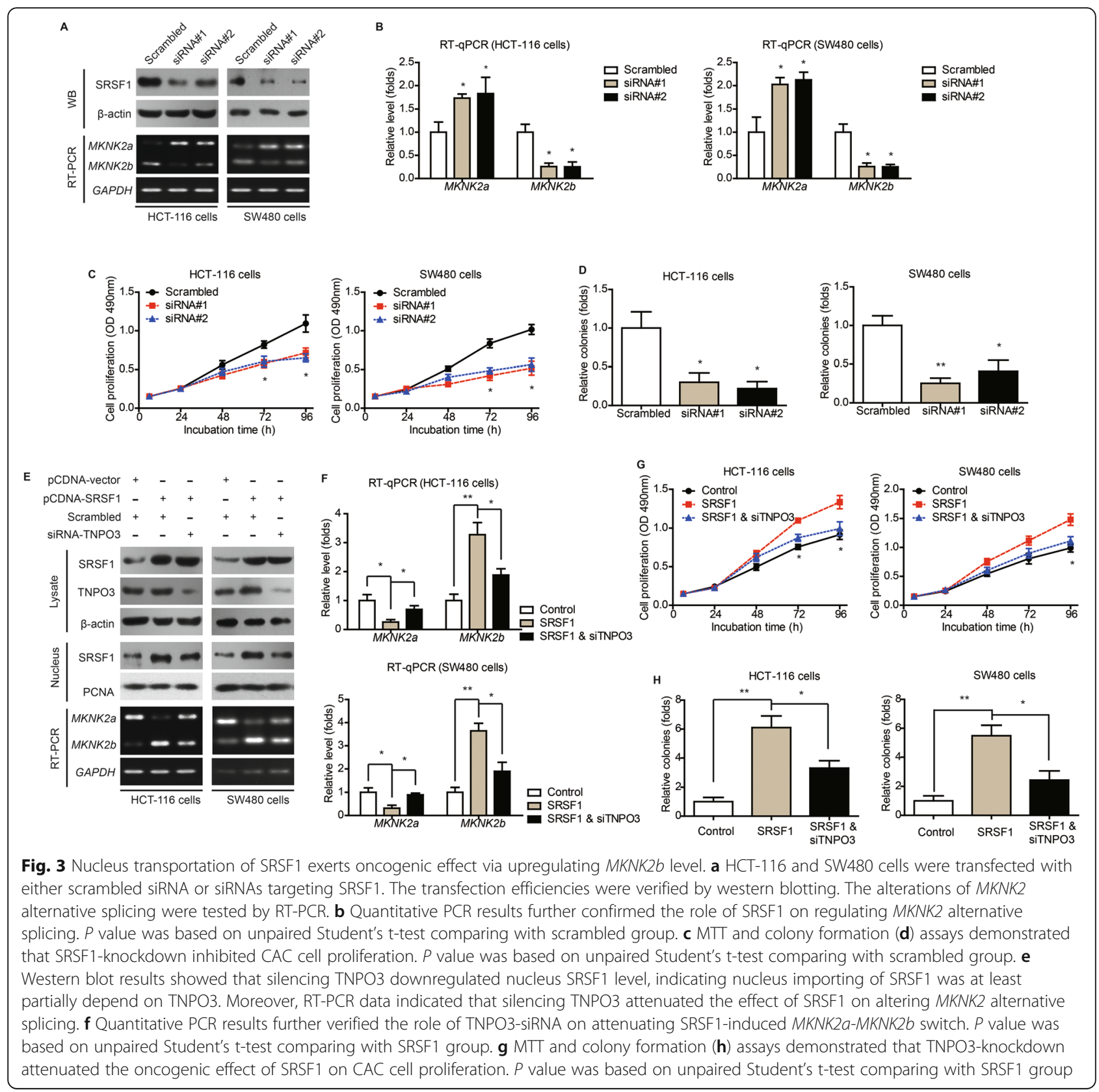

hypothesize that nucleus transportation is critical for SRSF1-guided $M K N K 2 a / M K N K 2 b$ switch. Previous studies had revealed that transportin 3 (TNPO3) can assist nucleus trafficking of SRSF members [18], thus we blocked the nucleus transportation of SRSF1 by silencing TNPO3 (Figure S3E). Accordingly, TNPO3-siRNA abolished the role of SRSF1 on promoting MKNK2a-MKNK2b switch (Fig. 3e, f) and resulted in attenuated cell proliferation (Fig. $3 \mathrm{~g}, \mathrm{~h}$ ).

SRPK1 and SRPK2 are positively correlated with MKNK2aMKNK2b switch and SRSF1 nucleus translocation Since phosphorylation plays vital roles on intracellular trafficking of SRSF proteins [19], we were interested to identify the upstream kinases responsible for SRSF1 nucleus transportation. CDC2-like kinase 1 (CLK1) and SR-specific protein kinases (SRPKs) had been proved to phosphorylate the RS domain of SRSF1 through in vitro kinase assays [20]. Therefore, we tested the statistical relevance between $M K N K 2 a-M K N K 2 b$ switch and CLK1, SRPK1, SRPK2, respectively. Correlation test revealed that CLK1 has no significant correlation with $M K N K 2$ alternative splicing (Fig. S4A). In contrast, the level of SRPK1 mRNA is positively correlated with $M K N K 2 b$ level, while negatively correlated with $M K N K 2 a$ level or $M K N K 2 a / M K N K 2 b$ ratio (Figure S4B). As for SRPK2, although no significance was 
observed towards $M K N K 2 a$ level, it showed a significant correlation with $M K N K 2 b$ (Fig. S4C). Besides, both SRPK1 and SRPK2 mRNA levels were upregulated in tumor tissues comparing with adjacent tissues in our primary cohort (Fig. 4a). TCGA database also revealed upregulated transcriptions of SRSF1 and SRPKs (Figure S5). Moreover, SRPK1 as well as SRPK2 showed positive correlations with nucleus-SRSF1 protein level (Fig 4b), indicating that SRPKs may promote nucleus translocation of SRSF1. We next evaluated protein expression level of SRPKs in primary cohort by immunoblotting. Consistent with mRNA data, both SRPK1 and SRPK2 proteins were elevated in tumor tissues (Fig. 4c).

\section{Prognostic effects of SRSF1, SRPK1, and SRPK2}

The findings in primary cohort and TCGA database promoted us to further investigate the prognostic significance of SRSF1 and SRPKs by introducing another independent validation cohort $(n=100)$. IHC experiments were conducted to explore the protein expression and localization. SRPK1 and SRPK2 were predominantly localized in cytoplasm, while SRSF1 was stained in both nucleus and cytoplasm (Fig. 4d). In accordance with primary cohort, CAC samples in validation cohort showed higher nucleus localization of SRSF1 and higher expression of SRPK1/2 (Fig. 4e). Besides, patients with higher SRPK1 or SRPK2 were characterized with higher nucleus-SRSF1 level (Fig. 4f). Furthermore, higher SRPK1 was observed in patients with larger tumor size and advanced tumor stages (Table S3).

Kaplan-Meier survival analyses revealed that patients with higher nucleus-SRSF1 had a poorer overall survival (Fig. 4g). Higher SRPK1 or higher SRPK2 also indicated unfavorable prognosis, respectively (Fig. $4 \mathrm{~h}$, i). Of note, patients with both high SRPK1 and high SRPK2 showed a lowest overall survival time comparing with other subgroups (Fig. 4j). Prognostic effects of other clinicopathological factors were also summarized, which demonstrated the clinical significance of lymph node metastasis and TNM stage (Fig. 4k, Table S4). Additionally, multivariate analysis was performed to explore independent prognostic variables (Fig. 4l, Table S5). Higher nucleus-SRSF1, higher SRPK1/2, and advanced TNM stage were all identified as independent risk factors of CAC overall survival.

\section{Restoration of Mnk2b reverses the anti-proliferation effects of SRPK knockdown}

The effect of SRPKs on CAC progression were validated by cellular experiments. The nonspecific SRPK inhibitor, SRPIN340, can significantly inhibit cell proliferation and colony formation of CAC cells (Fig. 5a, b). After testing the transfection efficiency of SRPK1-siRNAs and SRPK2-siRNAs (Figure S6A, S6B), we knocked down the two kinases in CAC cells and found that silencing either SRPK1 or SRPK2 showed no statistically significant effect on cell proliferation. However, simultaneously knockdown of both SRPK1 and SRPK2 remarkably attenuated cell proliferation and colony formation (Fig. 5c, d). Interestingly, overexpressing Mnk2b restored the cell proliferation capacity of SRPK1/2-silenced cells (Figure S6C-E). In contrast, SRPK1 and SRPK2 overexpression enhanced cell proliferation while TNPO3-siRNA almost abolished this effect (Figure S6F-H).

\section{SRPK1 and SRPK2 regulate MKNK2 alternative splicing by directly phosphorylating SRSF1 and promoting SRSF1 nucleus transportation}

We also compared the effects of SRPK1/2 knockdown or SRPK1/2 overexpression on SRSF1 phosphorylation, nucleus shuttling, and $M K N K 2$ alternative splicing in CAC cell lines (Fig. 5e, f; Figure S7A-D). As expected, either SRPK1-siRNA or SRPK2-siRNA significantly inhibited SRSF1 phosphorylation and nucleus translocation. In contrast, SRPK1 or SRPK2 overexpression upregulated the phosphorylation and nucleus accumulation of SRSF1. Besides, overexpressing SRPK1/2 led to an enhanced $M K N K 2 a-M K N K 2 b$ switch, while silencing SRPK1/2 showed opposite effects according to RT-PCR and RT-qPCR results. To further investigate whether kinase activity of SRPK1/2 were critical for SRSF1guided MKNK2 alternative splicing, we generated the kinase-dead mutants SRPK1-K109A and SRPK2-K110A. Either kinase-dead mutant or inhibitor SRPIN340 significantly attenuated, if not abolished, the role of SRPK1/2 on modulating SRSF1 phosphorylation, nucleus transportation as well as downstream $M K N K 2$ alternative splicing (Fig. 5g, h; Figure S7E-H). Moreover, immunoprecipitation data identified the intracellular interaction between SRSF1 with SRPK1/2 (Fig. 5i). Extracellular GST pull down assay was then conducted and confirmed their direct binding activity (Fig. 5j). We next investigate whether the interaction between SRPK1/2 and SRSF1 is a kinase-substrate process. In vitro phosphorylation test showed that both SRPK1 and SRPK2 are capable of phosphorylating SRSF1, which can be inhibited by SRPIN340 (Fig. 5k). Therefore, SRPK1 and SRPK2 may directly phosphorylate SRSF1 and promote it nucleus translocation, subsequently modulate MKNK2 alternative splicing.

\section{PP1a activity is downregulated in colon adenocarcinoma tissues}

It has been reported that $\mathrm{PP} 1 \alpha$ is the major phosphatase that dephosphorylates SRSF1 [21], thus we explored whether PP1 $\alpha$ is responsible for antagonizing SRPK $1 / 2$ during CAC development. To our surprise, no significant difference of PPP1CA mRNA level was observed 

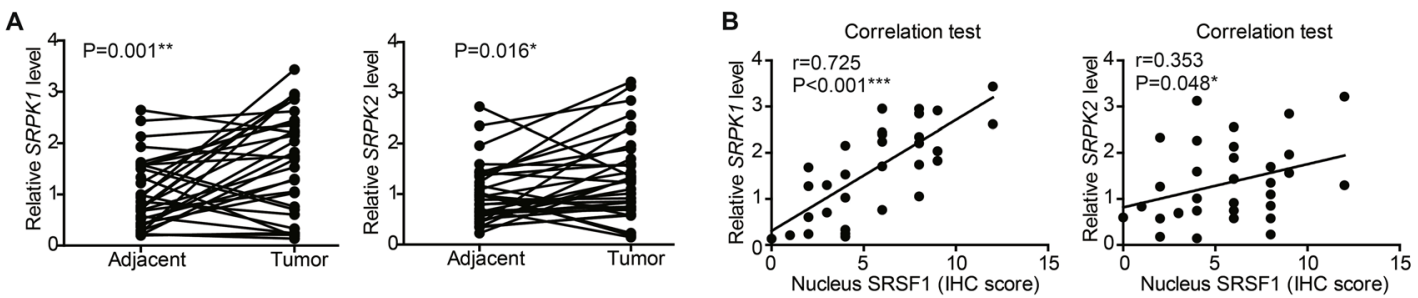

C
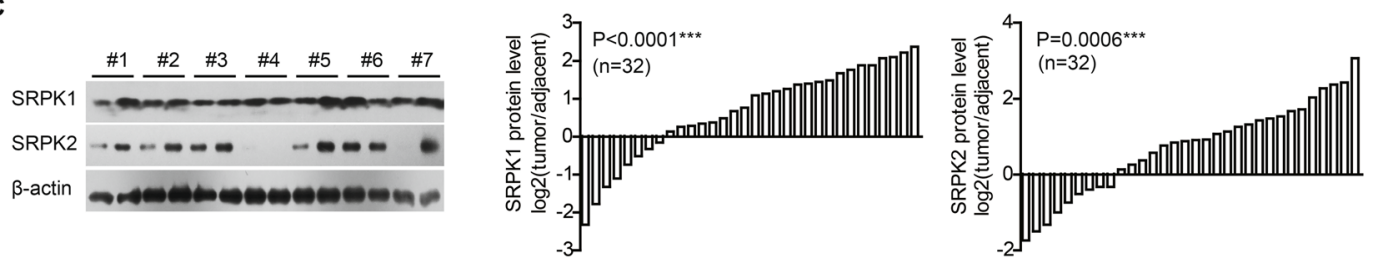

D
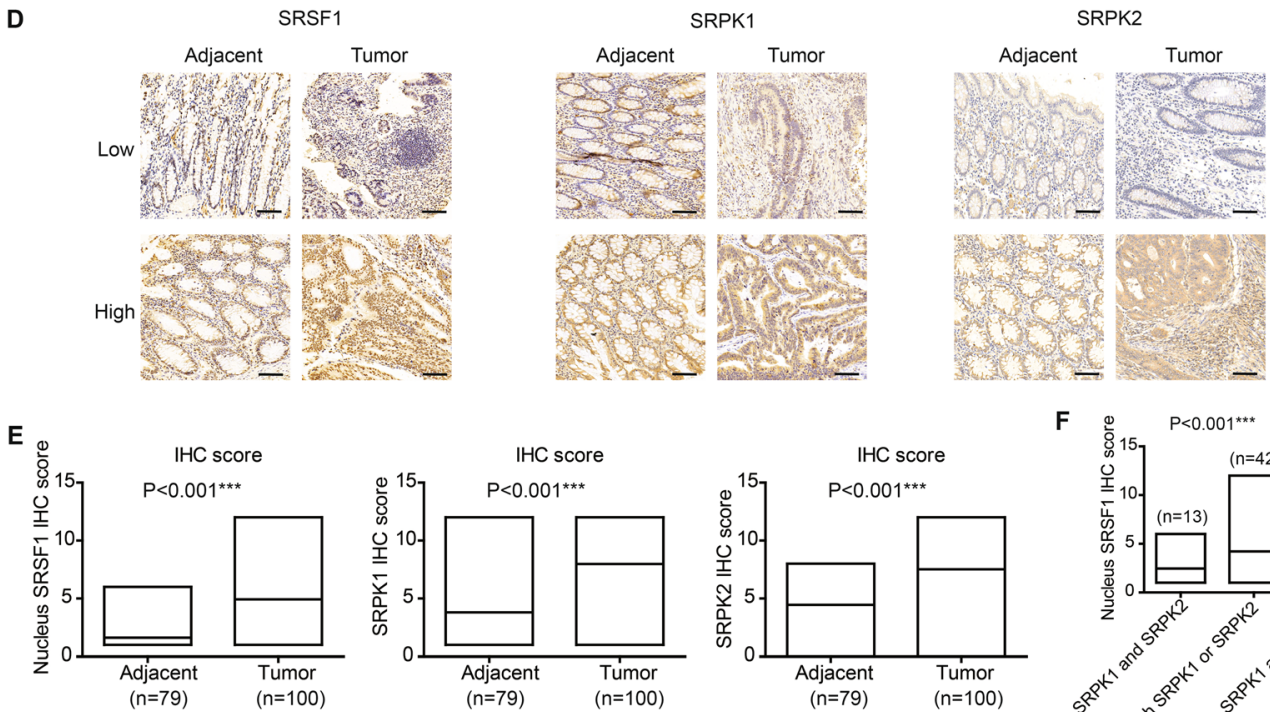

G
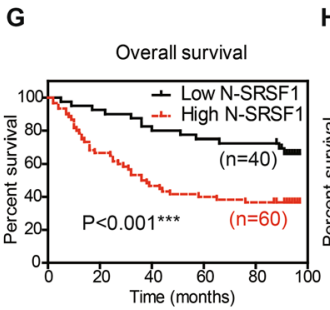

H

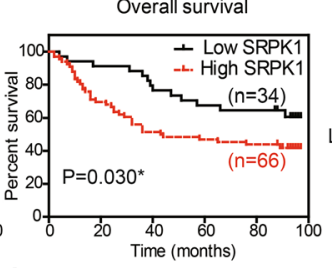

Overall survival
I
0

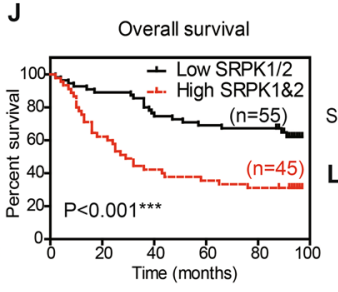

K

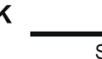

Univariable risk factor Sex (Male vs Female) Age (>68 vs $\leq 68 \mathrm{yrs}$ ) Pathological grade (II-III vs I) Size (>5.0 vs $\leq 5.0 \mathrm{~cm}$ ) Loacation (Ascending/Transverse vs Descending/Sigmoid) T stage (T4 vs T1-T3) $\mathrm{N}$ stage (N1-N2 vs NO) TNM stage (IIB-IIIC vs I-IIA) Nucleus SRSF1 (High vs Low) SRPK1 (High vs Low) SRPK2 (High vs Low) SRPK1 and SRPK2 (High vs Low)

$$
\text { L }
$$

Multivariable risk factor

Pathological grade (II-III vs I)TNM stage (IIB-IIIC vs I-IIA)Nucleus SRSF1 (High vs Low)SRPK1 and SRPK2 (High vs Low) $(n=100)$
F $\stackrel{\mathrm{O}}{\mathrm{O}}<0.001^{* * *}$

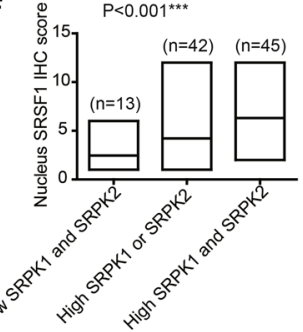

Fig. 4 (See legend on next page.) 
(See figure on previous page.)

Fig. 4 Expression and prognostic role of SRPKs in CAC. a RNA levels of upstream kinases targeting splicing factors in paired clinical specimens were tested by RT-qPCR $(n=32)$. P value was based on paired Student's t-test. $\mathbf{b}$ Correlations between nucleus SRSF1 level and upstream kinases were analyzed via Spearman correlation test $(n=32)$. The nucleus SRSF1 level was scored by the specific nucleus staining of SRSF1 (ranging 0-12) without considering its cytosol staining. c Protein levels of SRPK1 and SRPK2 in paired clinical specimens were tested by western blotting (left panel). After semi-quantified, we found that 75\% cases (24/32) showed increased SRPK1 in tumor tissues (middle panel), and 71.9\% cases (23/32) exhibited increased SRPK2 (right panel). d Representative IHC results of SRSF1, SRPK1, and SRPK2 in specimens from validation cohort. Scale bar:

$100 \mu \mathrm{m}$. e The different protein levels of SRPK1, SPRK2, and nucleus SRSF1 in validation cohort were exhibited by box plots according to IHC data. $P$ value was based on unpaired Student's t-test. $\mathbf{f}$ Patients with both high-SRPK1 and high-SRPK2 levels exhibited the highest nucleus SRSF1 level, while patients with low SRPK1 and low SRPK2 showed the lowest nucleus SRSF1 IHC score. $P$ value was based on One-way ANOVA test. KaplanMeier survival curves showed the clinical relevance of nucleus SRSF1 (g), SRPK1 (h), and SRPK2 (i) in validation cohort of CAC patients, respectively. Furthermore, the higher expression of both SRPK1 and SRPK2 showed a more significant role on indicating poorer overall survival (j). Forest plots summarized the significant prognostic factors by univariate $(\mathbf{k})$ and multivariate $(\mathbf{I})$ analyses, the corresponding data were supplemented in Table S4 and Table S5. P value was calculated by log-rank test or Cox-regression test

between tumor tissues and adjacent tissues (Fig. 6a). Taking into consideration that the phosphatase activity of PP1 $\alpha$ is as important as its expression level, we next tested whether the activity of PP1 $\alpha$ was altered in CAC tissues by testing its phosphorylation on T320 site, which inhibits its catalyzation capacity. IHC data showed cytoplasmic localization of both PP1 $\alpha$ and pT320-PP $1 \alpha$ (Fig. 6b). Consistent with RT-qPCR data, PP1 $\alpha$ protein level showed no difference between tumor and adjacent tissues. However, the pT320-PP1 $\alpha$ level was upregulated in tumor tissues, indicating a decreased PP1 $\alpha$ activity (Fig. 6c).

\section{PP1a negatively modulates SRSF1 nucleus transportation, MKNK2 alternative splicing, and tumor proliferation}

Furthermore, patients with higher nucleus-SRSF1 showed higher pT320-PP1 $\alpha$ level but not total PP1 $\alpha$ level (Fig. 6d; Figure S8A). Spearman correlation test also revealed a positive correlation between pT320-PP1 $\alpha$ and nucleus-SRSF1 (Fig. 6e) while no correlation was observed regarding total PP1 $\alpha$ (Figure S8B). Similarly, although no association was identified between total PP1 $\alpha$ and MKNK2 alternative splicing (Figure S8C), pT320$\mathrm{PP} 1 \alpha$ was positively correlated to $M K N K 2 a-M K N K 2 b$ switch (Fig. 6f).

In addition, the constitutively active mutant of $\mathrm{PP} 1 \alpha$, PP1 $\alpha-T 320 A$, significantly inhibited proliferation of CAC cells (Fig. 6g-i). Cellular assays were then performed to test crosstalk between PP1 $\alpha$ and SRSF1 by overexpressing constitutively active mutant (T320A) or inactive mutant $(\mathrm{H} 125 \mathrm{~A})$ of $\mathrm{PP} 1 \alpha$, respectively. We found that either PP1 $\alpha-\mathrm{WT}$ or PP $1 \alpha-\mathrm{T} 320 \mathrm{~A}$ can decrease SRSF1 phosphorylation and nucleus translocation, while PP1 $\alpha-\mathrm{H} 125 \mathrm{~A}$ showed opposite effects (Fig. 6j; Figure S8D). Consistently, PP1 $\alpha$-T320A inhibited $M K N K 2 a-M K N K 2 b$ switch while PP1 $\alpha-\mathrm{H} 125 \mathrm{~A}$ promoted it (Fig. 6j; Figure S8D). The PP1 $\alpha$ specific inhibitor, tautomycetin, can efficiently block the effects of PP $1 \alpha-$ WT or PP1 $\alpha-$ T320A on altering SRSF1 phosphorylation, nucleus translocation and $M K N K 2$ alternative splicing (Fig. 6k; Figure S8E). Furthermore, we observed similar findings on SW620 cells, the metastatic cell line of SW480 (Figure S9). Therefore, we concluded that PP1 $\alpha$ can suppress CAC progression at least partially by inhibiting SRSF1-guided $M K N K 2 a-M K N K 2 b$ switch, which is dependent on SRSF1 phosphorylation and nucleus shuttling.

\section{SRPKs and PP1a have opposite effects on modulating MKNK2 alternative splicing and tumor growth in mice model}

Xenograft models were utilized to verify tumor-related roles of SRPK1/2 and PP1 $\alpha$ in vivo. SW480 cells that stably overexpressed SRPK1\&2 or PP1 $\alpha$, or stably silenced SRPK1/2 with shRNAs were seeded subcutaneously in Balb/c nude mice. There was no statistical difference among the groups on the aspects of body weight nor food consumption (Fig. 7a, b). Among the four groups, SRPK1\&2-overexpressing group showed the highest proliferation capacity and largest tumor size. In contrast, PP $1 \alpha-\mathrm{T} 320 \mathrm{~A}$ group and sh-SRPK $1 / 2$ group both showed impaired growth process comparing to control group (Fig. 7b-e).

The mRNA levels of $M K N K 2 a$ and $M K N K 2 b$ in isolated xenografts were further tested by RT-qPCR, which revealed consistent results with cellular assays. On one hand, SRPK1\&2 downregulated $M K N K 2 a$ splicing and upregulated $M K N K 2 b$ level. On the other hand, PP1 $\alpha$ T320A or sh-SRPK1/2 attenuated MKNK2a-MKNK2b switch (Fig. 7f). Proliferation index of xenografts was also evaluated by calculating Ki-67 positive staining (Fig. $7 \mathrm{~g})$. As a result, SRPK1\&2 group showed the highest Ki67 staining percentage, while PP1 $\alpha-T 320 \mathrm{~A}$ group and sh-SRPK1/2 group showed decreased Ki-67 level, which is consistent with tumor growth curve (Fig. 7h). Taken together, our data revealed an oncogenic role of SRPK1/ 2 signaling in colon adenocarcinoma cells (Fig. 7i). In nontumorous cell, $M K N K 2$ pre-mRNA is predominantly spliced into $M K N K 2 a$ and translated to Mnk2a protein, which exerts anti-tumor effects. In colon 


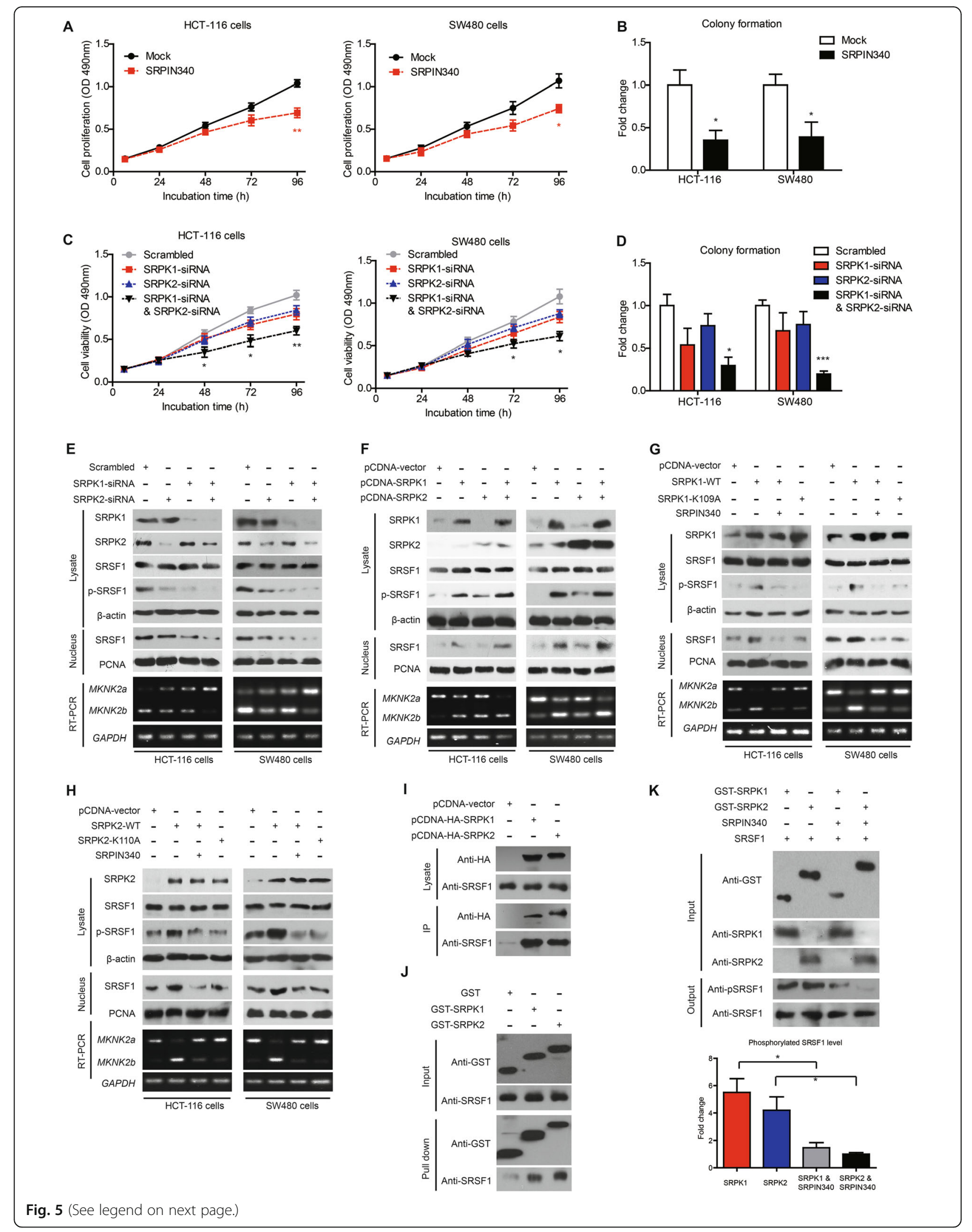


(See figure on previous page.)

Fig. 5 SRPKS promote SRSF1 phosphorylation, nucleus transportation, and downstream MKNK2 alternative splicing. a Treatment with SRPKs inhibitor, SRPIN340, resulted in decreased proliferation of HCT-116 and SW480 cells. P value was based on unpaired Student's t-test comparing with mock group which was treated with DMSO. b Colony formation assay also demonstrated an anti-proliferation effect of SRPIN340 on CAC cells, indicating the oncogenic role of SRPKs. $P$ value was based on unpaired Student's t-test comparing with mock group. c MTT and colony formation (d) assays demonstrated that silencing either SRPK1 or SRPK2 may impair CAC proliferation, although the statistical difference was not significant. Knockdown of both SRPK1 and SRPK2 can significantly inhibit CAC cell proliferation. $P$ value was based on unpaired Student's t-test comparing with scrambled group. e Knockdown of either SRPK1 or SRPK2 attenuated phosphorylation of SRSF1 (p-SRSF1) and reduced nucleus SRSF1 (N-SRSF1) level. RT-PCR results showed a positive correlation between MKNK2b and SRPKs. Furthermore, simultaneously knockdown of SRPK1 and SRPK2 exhibited the most significant effects. Semi-quantification of immunoblotting results and corresponding RT-qPCR data were shown in Supplementary Figure S7A-B. f Overexpressing SRPKs increased phosphorylation of SRSF1 and promoted its nucleus translocation according to immunoblotting results. Semi-quantification of immunoblotting results and corresponding RT-qPCR data were shown in Supplementary Figure S7C-D. g SRPIN340 treatment towards SRPK1-overexpressing cells abolished the effects above. Consistently, the kinase dead (KD) mutant SRPK1-K109A, lost effects on modulating SRSF1 phosphorylation, subcellular location, and MKNK2a-MKNK2b switch. Semiquantification of immunoblotting results and corresponding RT-qPCR data were shown in Supplementary Figure S7E-F. $\mathbf{h}$ SRPIN340 treatment towards SRPK2-overexpressing cells also abolished its effects on modulating SRSF1 phosphorylation, subcellular location, and MKNK2a-MKNK2b switch. The KD mutant of SRPK2, namely SRPK2-K110A, showed similar effects with SRPIN340. Semi-quantification of immunoblotting results and corresponding RT-qPCR data were shown in Supplementary Figure S7G-H. i SW480 cells were transfected with HA-tagged SRPK1 (pCDNA-SRPK1) or HA-tagged SRPK2 (pcDNA-SRPK2) or pcDNA-vector constructs. After lysing cells and conducting immunoprecipitation assay with anti-HA agarose, we found that SRPK1 and SRPK2 can interact with SRSF1 in CAC cells. $\mathbf{j}$ Purified GST-tagged SRPK1 or GST-SRPK2 proteins were incubated with purified SRSF1 proteins in vitro using GST protein as negative control. GST pull-down assay demonstrated the direct interaction between SRPK1/2 with SRSF1. The input was shown in upper panel. $\mathbf{k}$ The kinase activity of SRPK1/2 targeting SRSF1 was tested by in vitro kinase assay. Purified SRSF1 was incubated with GST-SRPK1 or GST-SRPK2 with/without SRPIN340 (10 $\mu \mathrm{M})$ as described in the Method section. Reaction products were separated via SDS-PAGE and analyzed by western blotting. Phosphorylation of SRSF1 in reaction products was semi-quantified after normalized by total SRSF1 (lower panel). The data showed that both SRPK1 and SRPK2 can directly phosphorylate SRSF1, which can be inhibited by the inhibitor SRPIN340

adenocarcinoma cells, SRSF1 is hyperphosphorylated due to elevated SRPK1/2 and decreased PP1 $\alpha$ activity, resulting in SRSF1 nucleus shuttling. Nucleus SRSF1 accumulation finally leads to alterative splicing of $M K N K 2$ pre-mRNA to $M K N K 2 b$ and upregulated the Mnk2b oncoprotein level (Fig. 7i).

\section{Discussion}

Alternative splicing expands the proteomic complexity by generating splicing variants on mRNA level and encoded distinct protein isoforms including kinases. In the present study, we focused on $M K N K 2 a$ and $M K N K 2 b$ alternative splicing, encoding Mnk2a and Mnk2b, respectively. Our data revealed a downregulation of $M K N K 2 a$ and an upregulation of $M K N K 2 b$ in colon adenocarcinomas, which is closely correlated with KRAS mutation. The hypersynthesis of $M K N K 2 b$ is observed in samples with larger tumor size, implying its role on promoting tumor proliferation. Maimon et al. had recently reported that Mnk2b is deficient on activating p38-induced cell death pathway, while maintaining its capacity of promoting eIF4Einduced oncoprotein translation. In contrast, Mnk2a can induce both oncogenesis as well as p38-MAPK stress apoptosis pathway. Therefore, they elucidated downstream mechanism of how Mnk2a and Mnk2b exert opposite effects on tumor progression in Ras-activated cells [10]. Consistently, our data also revealed a significant correlation between $M K N K 2$ splicing shift with KRAS mutation in colon adenocarcinomas, while no obvious association with TP53 was identified. Additionally, cell lines possessing KRAS mutation showed a prevalence of $M K N K 2 a-M K N K 2 b$ switch in comparison with KRASWT cell lines. Strikingly, KRAS-G12V transfection enhanced $M K N K 2 b$ mRNA splicing even in nontumorous colon epithelial cells, indicating an oncogenesis correlation between KRAS mutation and MKNK2 alternative splicing. According to the data reported by Maimon et al. [10], oncogenic transformation caused by activated Ras can induce $M K N K 2 a-M K N K 2 b$ switch in breast cancer cells, pancreatic cancer cells and in lung cancer cells. Furthermore, coexpressing Mnk2a inhibited the oncogenic phenotype of Ras-transformed MCF-10A cells, indicating that Mnk2a can block Ras-induced transformation [10]. Therefore, it is high likely that oncogenic Ras induces tumor transformation at least partially through affecting $M K N K 2 a-M K N K 2 b$ switch. Another study by Maimon et al. artificially induced a strong switch from $M K N K 2 b$ to $M K N K 2 a$ by using designated oligonucleotides, resulting in inhibited glioblastoma development and resensitization to chemotherapy [22]. Their study provides evidence on the therapeutic potential of manipulating $M K N K 2$ alternative splicing as a novel approach to treat glioma. Our present results regarding $M K N K 2 a-M K N K 2 b$ switch in colon adenocarcinoma implicated that similar intervention may be a novel direction for CAC treatment.

In addition, our RT-qPCR screening assay indicated SRSF1 as a promising modulator targeting $M K N K 2$ alternative splicing, which was confirmed by correlation test, RNA immunoprecipitation, and knockdown assays. The findings on that nuclear SRSF1, instead 


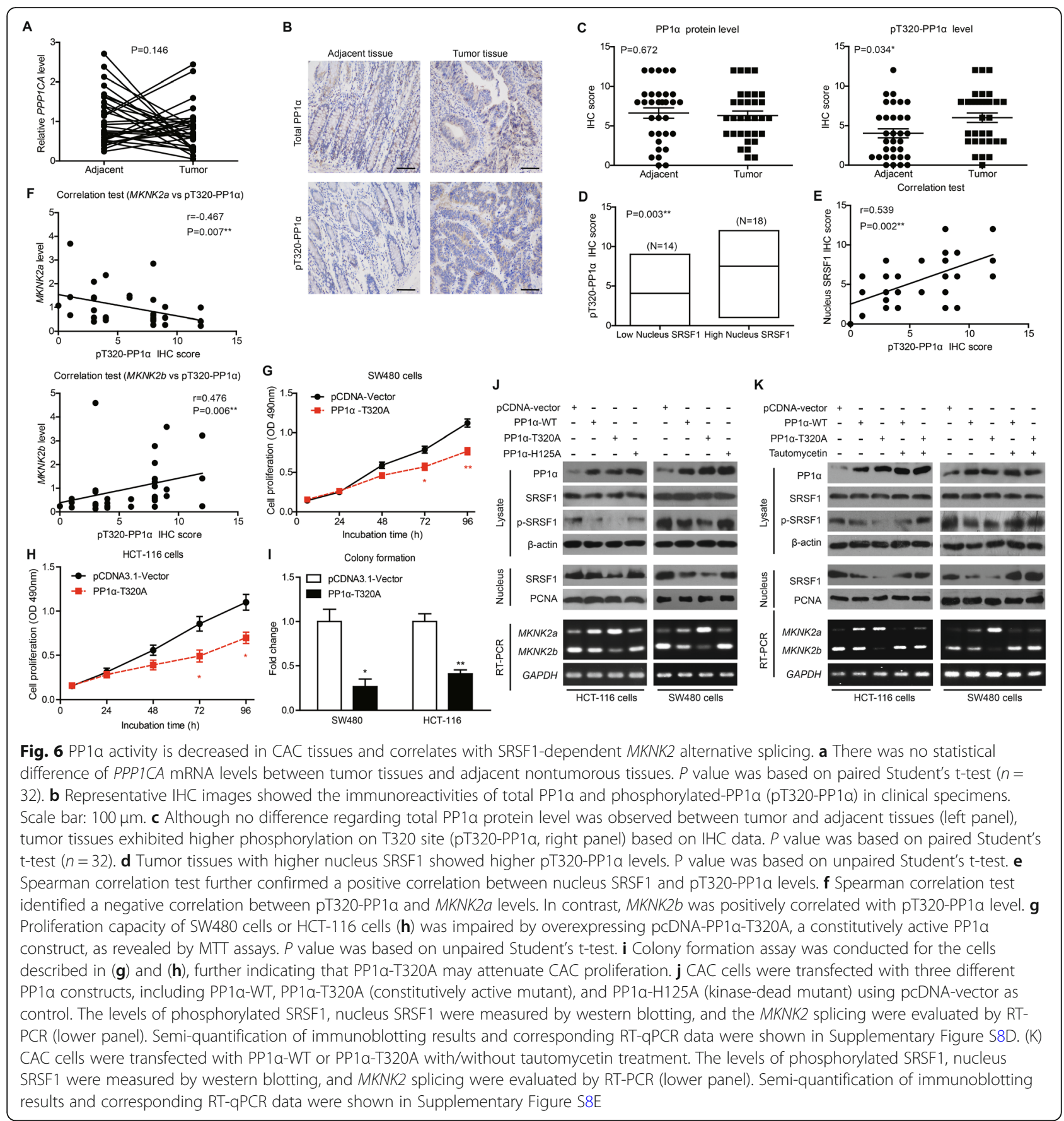

of cytoplastic SRSF1, was correlated with patients' clinical outcomes promoted us to further explore the upstream regulation mechanisms of SRSF1 subcellular trafficking. Consistent with electron microscopy data [23], we verified the critical role of TNPO3 on importing SRSF1 into nucleus of colon adenocarcinoma cells. Further study showed that the tumorpromoting role of SRSF1 can be significantly attenuated by silencing TNPO3, confirming that only nucleus SRSF1 exerts pro-oncogenic effects.
Post-translational modification has remained the focus of protein function investigation during the past decades, and phosphorylation is one of the most important modifications which we focused on [24, 25]. Abnormal expression of kinases or phosphatases can result in dysregulated phosphorylation events, leading to various malignancies as we previously reported $[14,26]$. It has been reported that multisite phosphorylation of SRSF1 is a prerequisite for its nucleus transportation [27, 28], which is the key mechanism for its functional alternative 


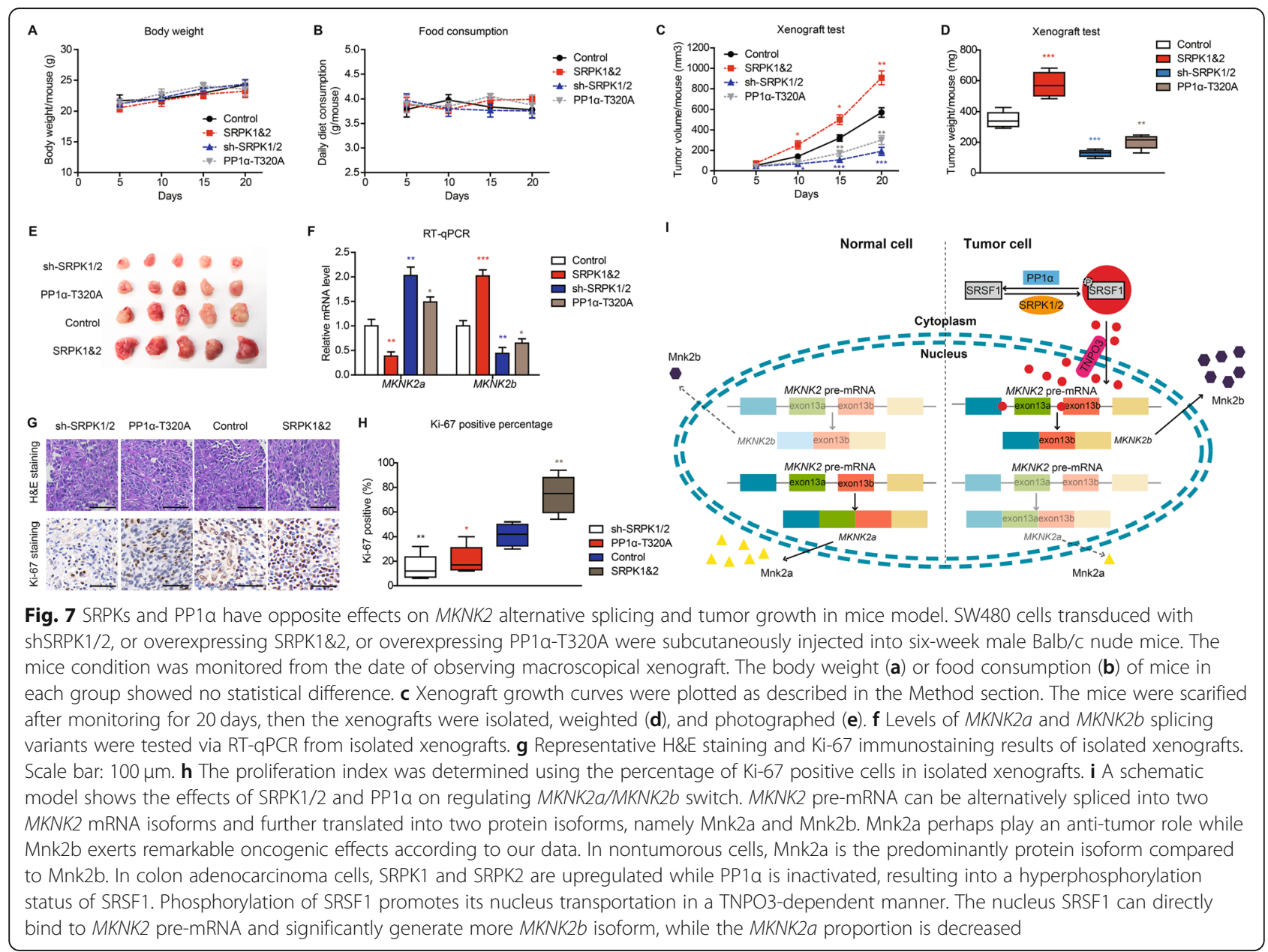

splicing. Therefore, we next aimed to investigate the upstream kinase and phosphatase which controls SRSF1 phosphorylation. Three major kinases had been reported to phosphorylate SRSF1 including SRPK1, SRPK2, and CLK1 $[19,20]$. Considering that enzyme-substrate relationship can be difference in various cell types, we initially explored whether those kinases could affect SRSF1 nucleus translocation in colon adenocarcinoma cells. Although we didn't observe any statistical correlation between CLK1 and nucleus SRSF1 level, both SRPK1 and SRPK2 indeed correlated with SRSF1 nucleus translocation. Additionally, SRPK1 and SRPK2 showed upregulated expression in CAC tissues compared with adjacent tissues, which is consistent with their expression patterns in acute myeloid leukemia, breast cancer, and pancreatic cancer [29-31].

In accordance with their underlying crosstalk, SRPK1/ 2 and SRSF1 are all identified as independent risk factors for the overall survival of CAC patients. Following cellular results again confirmed that silencing SRPK1 and SRPK2 can lead to decreased SRSF1 phosphorylation, nucleus transportation, MKNK2a-MKNK2b switch, and cell proliferation. Similar results were obtained by introducing kinase-dead mutants or using their inhibitor SRPIN340. Of note, we systematically verified that both SRPK1 and SRPK2 can directly interact and phosphorylate SRSF1 by immunoprecipitation, GST pull-down, and in vitro phosphorylation test. The anti-tumor effects of SRPK inhibition had been recently reported in other malignancies [31, 32], our data enlarged the therapeutic potential of developing SRPK inhibitor for CAC treatment. Interestingly, a previous study by Wang et al. demonstrated that SRPK1 can act as both oncogene and tumor suppressor, either higher or lower level of SRPK1 induces cell transformation [33]. The underlying mechanism is determined by the interaction between SRPK1 and PHLPP, thus aberrant SRPK1 expression in either direction could result in hyperactivation of Akt by blocking its phosphatase PHLPP. Considering that specific effect of kinase is largely depend on its substrate, identification of other SRPK1 substrates will be invaluable for further illustrating its tumor-related role. Anyway, here our data confirmed the pro-oncogenic axis of SRPK1/2-SRSF1-MKNK2 in CAC progression. 
Besides kinases, we next focused on SRSF1 upstream phosphatase to impartially evaluate the importance of its phosphorylation in CAC. Adams JA et.al and her colleagues reported that protein phosphatase $1 \alpha(\mathrm{PP} 1 \alpha)$ directly dephosphorylates the RS domain of SRSF1 through binding to its RNA recognition motif 1 (RRM1) through an allosteric mechanism [34]. Of note, disrupting the interaction between PP1 $\alpha$ and SRSF1 results in significant shifts of alternative splicing such as EIF5 and TIMM8B genes [35]. On the other hand, PP1 $\alpha$ has been demonstrated to inhibit the metastasis of SW620 colon cancer cells via deactivating Src protein [36]. Therefore, we were promoted to assess whether PP1 $\alpha$ was dysregulated in colon adenocarcinoma. Although we didn't find any significant difference of $\mathrm{PP} 1 \alpha$ expression between CAC tissues and adjacent tissues, its catalytic inactivate type, namely the Tyr320-phosphorylated PP1 $\alpha$, was elevated in tumor tissues, indicating that $\mathrm{PP} 1 \alpha$ activity was downregulated in colon adenocarcinoma. Here we also validated the role of $\mathrm{PP} 1 \alpha$ on regulating $M K N K 2$ alternative splicing by introducing its constitutively active mutant and inactive mutant, respectively. Besides SW480 and HCT-116 cells, PP1 $\alpha$ and its mutations showed similar effects on the metastatic SW620 cell line, further highlighting the prognostic value of $P P 1 \alpha-$ SRSF1-MNK2 axis in CAC progression. However, we have to keep in mind that phosphorylation status of SR proteins can not only modulate their subcellular localization but also determines the splicing site selection [37], thus the more detailed effects of SRPKs and $\mathrm{PP} 1 \alpha$ on alternative splicing need further validation. Together with the findings from xenografts, we concluded that PP1 $\alpha$ can attenuates colon cancer progression at least partially via antagonizing SRPK1/2-induced SRSF1 phosphorylation and downstream MKNK2a-MKNK2b splicing shift.

\section{Conclusions}

Our study reveals a significant role of SRSF1-guided $M K N K 2 a-M K N K 2 b$ splicing switch in the proliferation of colon adenocarcinoma, which is determined by SRSF1 phosphorylation and subcellular translocation. The alternative splicing of MKNK2 is balanced by SRPK1/2 and $\mathrm{PP} 1 \alpha$, thus providing opportunities of therapeutic intervention, such as SRPK inhibitors or PP1 $\alpha$ allosteric activators, in treating malignancies. Additionally, the subcellular location of SRSF1, expression levels of SRPK1/2, as well as phosphorylation status of PP1 $\alpha$ can all serve as independent prognostic predictors for the overall survival of colon adenocarcinoma.

\section{Supplementary Information}

The online version contains supplementary material available at https://doi. org/10.1186/s13046-021-01877-y.
Additional file 1: Table S1. Patients information of primary cohort. Table S2. Correlations between MKNK2 alternative splicing and splicing factors on mRNA levels. Table S3. Correlation between protein expression level and clinical features of colon adenocarcinoma patients in validation cohort. Table S4. Kaplan-Meier survival analyses for colon adenocarcinoma patients. Table S5. Cox-regression analysis for overall survival of colon adenocarcinoma patients. Table S6. Antibodies, chemicals, siRNAs, shRNAs, and primers in this study. Figure S1. Correlations between MKNK2 alternative splicing with patients' characteristics in primary cohort. Figure S2. Correlations between splicing factors and MKNK2 alternative splicing in CAC tissues. Figure S3. Correlations between SRSF1 protein expression and MKNK2 alternative splicing in CAC tissues. Figure S4. Correlations between upstream kinases and MKNK2 alternative splicing in CAC tissues. Figure S5. SRSF1, SRPK1, and SRPK2 are upregulated in CAC tissues. Figure S6. Effects of SRPKs on cell proliferation can be altered by Mnk2b and TNPO3. Figure S7. SRPKs modulate SRSF1 phosphorylation, nucleus transportation, and MKNK2 alternative splicing. Figure S8. Functions of PP1a on modulating SRSF1-dependent MKNK2 splicing. Figure S9. Functions of PP1a on modulating proliferation and SRSF1-dependent MKNK2 splicing in metastatic SW620 cells.

\section{Abbreviations}

CAC: Colon adenocarcinoma; CLK1: CDC2-like kinase 1; elF4E: Eukaryotic translation initiation factor 4E; Mnk: MAP kinase-interacting serine/threonineprotein kinase; PP1a: Protein phosphatase 1a; RNA-IP: RNA

immunoprecipitation; SRSF: Serine and arginine rich splicing factor; SRPK: SRspecific protein kinase; TNPO3: Transportin 3

\section{Acknowledgements}

We thank Jinpeng Sun (Shandong University) for providing purified GST proteins. We thank Xiangrong Kong (Qingdao Urban Planning \& Design Research Institute) for helpful discussion during manuscript preparation.

\section{Authors' contributions}

$\mathrm{HL}$ designed this project and analyzed the data. ZG help conduct xenograft experiments. KL conducted RT-PCR and RT-qPCR analyses. QZ performed cell proliferation tests and WB tests. ZX and $Y X$ were major contributors in interpreting the data and writing the manuscript. All authors read and approved the final manuscript.

\section{Funding}

Our study was supported by Fellowship of China Postdoctoral Science Foundation (Grant No. 2020 M671392), Fellowship of Jiangsu Postdoctoral Science Foundation (Grant No. 2020Z069), Special Foundation for National Science and Technology Basic Research Program of China (Grant No. 2019FY101104), National Natural Science Foundation of China (Grant No. 81601668), Shandong Province Major Research and Design Program (Grant No. 2018GSF118169), and Jinan City Science and Technology Development Program (Grant No.201805017,201805013).

\section{Availability of data and materials}

The data regarding the current study are available on reasonable request.

\section{Ethics approval and consent to participate}

All experiments were approved and supervised by the Ethics Committee of Qilu Hospital of Shandong University. Informed consent was obtained from all individual participants included in the study.

Animal studies in nude mice were approved by the Ethical Committee of Shandong University and carried out in accordance with the UK Animals (Scientific Procedures) Act, 1986.

Consent for publication

Not applicable.

Competing interests

None. 


\section{Author details}

'Department of General Surgery, The First Affiliated Hospital of Nanjing Medical University, 300 Guangzhou Road, Nanjing 210029, China. ${ }^{2}$ The Jackson Laboratory, Bar Harbor, ME 04609, USA. ${ }^{3}$ Department of General Surgery, Qilu Hospital of Shandong University, Jinan 250012, China. ${ }^{4}$ Department of Respiratory Medicine, The First Affiliated Hospital of Nanjing Medical University, Nanjing 210029, China.

\section{Received: 4 January 2021 Accepted: 11 February 2021}

Published online: 18 February 2021

\section{References}

1. Siegel RL, Miller KD, Jemal A. Cancer statistics, 2020. CA Cancer J Clin. 2020; 70:7-30.

2. Siegel RL, Miller KD. Colorectal cancer statistics. Multicenter Study. 2020;70: 145-64

3. Siegel RL, Miller KD, Jemal A. Cancer statistics, 2019. CA Cancer J Clin. 2019; 69:7-34.

4. Yang $X$, Zhong W, Cao R. Phosphorylation of the mRNA cap-binding protein elF4E and cancer. Cell Signal. 2020;73:109689.

5. Hou S, Du P, Wang P, Wang C, Liu P, Liu H. Significance of MNK1 in prognostic prediction and chemotherapy development of epithelial ovarian cancer. Clin Transl Oncol. 2017;19:1107-16.

6. Guo Z, Peng G, Li E, Xi S, Zhang Y, Li Y, et al. MAP kinase-interacting serine/ threonine kinase 2 promotes proliferation, metastasis, and predicts poor prognosis in non-small cell lung cancer. Sci Rep. 2017;7:10612.

7. Xie J, Shen K. Reciprocal signaling between mTORC1 and MNK2 controls cell growth and oncogenesis. Cell Mol Life Sci. 2020;78(1):249. https://doi. org/10.1007/s00018-020-03491-1.

8. Scheper GC, Parra JL, Wilson M, Van Kollenburg B, Vertegaal AC, Han ZG, et al. The $\mathrm{N}$ and $\mathrm{C}$ termini of the splice variants of the human mitogenactivated protein kinase-interacting kinase Mnk2 determine activity and localization. Mol Cell Biol. 2003:23:5692-705.

9. Stead RL, Proud CG. Rapamycin enhances elF4E phosphorylation by activating MAP kinase-interacting kinase 2a (Mnk2a). FEBS Lett. 2013;587: 2623-8.

10. Maimon A, Mogilevsky M, Shilo A, Golan-Gerstl R, Obiedat A, Ben-Hur V, et al. Mnk2 alternative splicing modulates the p38-MAPK pathway and impacts Ras-induced transformation. Cell Rep. 2014;7:501-13.

11. Zhang Q, Fan H, Liu H, Jin J, Zhu S, Zhou L, et al. WNT5B exerts oncogenic effects and is negatively regulated by miR-5587-3p in lung adenocarcinoma progression. Oncogene. 2020;39:1484-97.

12. Liu H, Liu Z, Li K, Li S, Song L, Gong Z, et al. TBL1XR1 predicts isolated tumor cells and micrometastasis in patients with TNM stage I/II colorectal cancer. J Gastroenterol Hepatol. 2017;32:1570-80.

13. Zhang XM, Liu ZL, Qiu B, Xu YF, Pan C, Zhang ZL. Downregulation of EVI1 expression inhibits cell proliferation and induces apoptosis in Hilar Cholangiocarcinoma via the PTEN/AKT Signalling pathway. J Cancer. 2020; 11:1412-23.

14. Xu YF, Liu ZL, Pan C, Yang XQ, Ning SL, Liu HD, et al. HMGB1 correlates with angiogenesis and poor prognosis of perihilar cholangiocarcinoma via elevating VEGFR2 of vessel endothelium. Oncogene. 2019:38:868-80.

15. Yang F, Yu X, Liu C, Qu CX, Gong Z, Liu HD, et al. Phospho-selective mechanisms of arrestin conformations and functions revealed by unnatural amino acid incorporation and (19) F-NMR. Nat Commun. 2015;6:8202.

16. Humeau J, Sauvat A, Cerrato G, Xie W, Loos F. Inhibition of transcription by dactinomycin reveals a new characteristic of immunogenic cell stress. EMBO Mol Med. 2020;12:e11622.

17. Zheng X, Peng Q, Wang L, Zhang X, Huang L, Wang J, et al. Serine/argininerich splicing factors: the bridge linking alternative splicing and cancer. Int J Biol Sci. 2020;16:2442-53.

18. Wan L, Yu W, Shen E, Sun W, Liu Y, Kong J, et al. SRSF6-regulated alternative splicing that promotes tumour progression offers a therapy target for colorectal cancer. Gut. 2019;68:118-29.

19. Long Y, Sou WH, Yung KWY, Liu H, Wan SWC, Li Q, et al. Distinct mechanisms govern the phosphorylation of different SR protein splicing factors. J Biol Chem. 2019;294:1312-27.

20. Aubol BE, Plocinik RM, Hagopian JC, Ma CT, McGlone ML, Bandyopadhyay R, et al. Partitioning RS domain phosphorylation in an SR protein through the CLK and SRPK protein kinases. J Mol Biol. 2013;425:2894-909.
21. Jang S-W, Liu X, Fu H, Rees H, Yepes M, Levey A, et al. Interaction of Aktphosphorylated SRPK2 with 14-3-3 mediates cell cycle and cell death in neurons. J Biol Chem. 2009;284:24512-25.

22. Mogilevsky M, Shimshon O, Kumar S, Mogilevsky A, Keshet E, Yavin E, et al. Modulation of MKNK2 alternative splicing by splice-switching oligonucleotides as a novel approach for glioblastoma treatment. Nucleic Acids Res. 2018;46:11396-404.

23. Costa R, Rodia MT, Zini N, Pegoraro V, Marozzo R, Capanni C, et al. Morphological study of TNPO3 and SRSF1 interaction during myogenesis by combining confocal, structured illumination and electron microscopy analysis. Mol Cell Biochem. 2021. https://doi.org/10.1007/s11010-020-04023y.

24. Xiao K, Liu H. "Barcode" and differential effects of GPCR phosphorylation by different GRKs; in G protein-coupled receptor kinases. Springer. 2016;1:75120.

25. Liu H, Wang D, Zhang Q, Zhao Y, Mamonova T, Wang $L$, et al. Parallel posttranslational modification scanning enhancing hydrogen-deuterium exchange-mass spectrometry coverage of key structural regions. Anal Chem. 2019;91:6976-80.

26. Qiu B, Chen T, Sun R, Liu Z, Zhang X, Li Z, et al. Sprouty4 correlates with favorable prognosis in perihilar cholangiocarcinoma by blocking the FGFRERK signaling pathway and arresting the cell cycle. EBioMedicine. 2019;50: 166-77.

27. Serrano P, Aubol BE, Keshwani MM, Forli S, Ma C-T, Dutta SK, et al. Directional phosphorylation and nuclear transport of the splicing factor SRSF1 is regulated by an RNA recognition motif. J Mol Biol. 2016;428:243045.

28. Aubol BE, Wu G, Keshwani MM, Movassat M, Fattet L, Hertel KJ, et al. Release of SR proteins from CLK1 by SRPK1: a symbiotic kinase system for phosphorylation control of pre-mRNA splicing. Mol Cell. 2016;63:218-28.

29. Tzelepis K, De Braekeleer E, Aspris D, Barbieri I, Vijayabaskar M, Liu W-H, et al SRPK1 maintains acute myeloid leukemia through effects on isoform usage of epigenetic regulators including BRD4. Nat Commun. 2018;9:5378.

30. Wang G, Sheng W, Shi X, Li X, Zhou J, Dong M. Serine/arginine proteinspecific kinase 2 promotes the development and progression of pancreatic cancer by downregulating numb and p53. FEBS J. 2019;286:1668-82.

31. Siqueira RP, Caetano MMM, de Souza L, Dos Passos PMS, Simaroli NB, Barros MVA, et al. Combined SRPK and AKT pharmacological inhibition is synergistic in T-cell acute lymphoblastic leukemia cells. Toxicol in Vitro. 2020;65:104777.

32. Hatcher JM, Wu G, Zeng C, Zhu J, Meng F, Patel S, et al. SRPKIN-1: a covalent SRPK1/2 inhibitor that potently converts VEGF from proangiogenic to anti-angiogenic isoform. Cell Chem Biol. 2018;25:460-470. e466.

33. Wang P, Zhou Z, Hu A, Ponte de Albuquerque C, Zhou Y, Hong L, et al. Both decreased and increased SRPK1 levels promote cancer by interfering with PHLPP-mediated dephosphorylation of Akt. Mol Cell. 2014;54:378-91.

34. Aubol BE, Hailey KL, Fattet $L$, Jennings PA, Adams JA. Redirecting SR protein nuclear trafficking through an allosteric platform. J Mol Biol. 2017;429:217891.

35. Aubol BE, Serrano P, Fattet L. Molecular interactions connecting the function of the serine-arginine-rich protein SRSF1 to protein phosphatase. J Biol Chem. 2018;293:16751-60.

36. Chen J. Is Src the key to understanding metastasis and developing new treatments for colon cancer? Nat Clin Pract Gastroenterol Hepatol. 2008;5: 306-7.

37. Jeong S. SR proteins: binders, regulators, and connectors of RNA. Mol Cell. 2017;40:1.

\section{Publisher's Note}

Springer Nature remains neutral with regard to jurisdictional claims in published maps and institutional affiliations. 